\section{Prädisposition, genetische}

\section{J. Arnemann}

Abteilung Molekulargenetik, Labor Dr. Wisplinghoff, Köln, Deutschland

Synonym(e) Erkrankungsanfälligkeit

Englischer Begriff genetic predisposition

Definition Genetische Prädisposition, ein Begriff aus der Medizinischen Genetik, beschreibt eine genotypische Anlage für einen bis dahin nicht ausgeprägten Phänotyp.

Beschreibung Bei Ratsuchenden wird oftmals eine potenziell pathogene Mutation im Genom nachgewiesen, die erst im Laufe der Entwicklung oder im vorangeschrittenen Lebens- alter sich als Krankheit ausprägen kann (sog. Late-onsetErkrankung). Für eine mögliche Erkrankung und auch Verlauf der Erkrankung sind vielfach multifaktorielle Einflüsse verantwortlich, wie zusätzliche genetische Varianten, Mutationslast (insbesondere bei erblichen Tumorerkrankungen) oder Lifestyle-Einflüsse.

Eine genetische Testung auf erbliche Mutationen ermöglicht beim Ratsuchenden eine gezielte genetische Beratung zu Krankheitsprognosen oder auch individuellen Präventionsmaßnahmen.

\section{Literatur}

Henn W., Zang K.D. (2006) Genetische Prädisposition. In: Schmoll, HJ., Höffken K., Possinger K. (eds) Kompendium Internistische Onkologie. Springer, Berlin, Heidelberg 